\title{
Fault Diagnosis for Rolling Bearing Based on Lifting Wavelet and Multi-fractal Dimension
}

\author{
Zhongyun ZHANG ${ }^{1, a}$, Jiande $W^{1,2, b}$, Jun $M^{1, b}$, Xiaodong WANG ${ }^{1,2, b}$ \\ ${ }^{1}$ Faculty of Information Engineering and Automation, Kunming University of Science and \\ Technology, Kunming, 650500, China \\ ${ }^{2}$ Engineering Research Center for Mineral Pipeline Transportation YN, Kunming, 650500, China \\ aemail:747677914@qq.com, 'bjiande@foxmail.com
}

\section{Keywords: Rolling Bearing; Fault Diagnosis; Lifting Wavelet; Multi-fractal Dimension}

\begin{abstract}
The vibration signal of rolling bearing is complex and nonstationary. In the process of fault diagnosis, if only describe the single fractal characteristics of signal, some certain conditions can't be identified accurately. Therefore, this paper uses the multi-fractal dimension as the characteristic quantity, puts forward a method based on lifting wavelet transform and multi-fractal dimension for rolling bearing fault diagnosis. The step of the diagnosis goes as follows: firstly, decompose the vibration signal of rolling bearing into three layers and reconstruct it by lifting wavelet transform, to highlight the state characteristics of vibration signal; Secondly, calculate the multi-fractal dimension of reconstructed signal, and take it as characteristic quantity to discriminate the bearing status; finally, the vibration signal of rolling bearing in each condition are selected for experimental comparison and analysis, to prove that the method is feasible.
\end{abstract}

\section{Introduction}

Rolling bearing is one of the most important and the most prone to failure components in rotating machinery. The bearing fault directly affect the proper function of equipment, so it is very important to have a careful diagnosis in the rolling bearing [1]. Fault feature extraction is the key to fault diagnosis of bearing. However, duo to the influence of transmission path and noise, the process of rolling bearing is a dynamic process which is complex and non-stationary, and whose vibration signal also exhibits strong nonlinear and non-stationary, greatly increase the difficulty of the rolling bearing fault diagnosis.

The fractal dimension is one of the effective methods for fault feature extraction in recent years. The fractal dimension has a good capability for represent nonlinear signal. Jin Ma et al [2] have applied the fractal theory to fault diagnosis of gas valves, calculate the correlation dimension of vibration signal and take it as the characteristics for the gas valve early fault diagnosis, it achieved good effect; What's more, the fractal dimension is also used to the fault diagnosis of internal combustion engines, sliding bearings, motors and so on [3] [4] [5]. But most of these applications are single fractal characteristics, it can only reflect the irregular degree of signal from the whole, can't accurately describe its local singularity. Study found that, the multi-fractal dimension can improve the finer levels of reflecting local scaling behavior, can describe the local scaling behavior of signal more precise [6]. In practice, the original vibration signal of rolling bearing usually with low SNR (noise-signal ratio) because of the effect of transmission path and noise. Thus, the result is not very reliable if extract the fractal feature of signal directly. In order to obtain the accurate multi-fractal dimension of signal, must do noise reduction first. Lifting wavelet method is the second generation of wavelet transform, Compared to the traditional wavelet, not only it maintains the advantages of traditional wavelet time-frequency localization characteristic, but also its speed is faster and more accurate [7]. Therefore, this paper puts forward the method of fault diagnosis for rolling bearing based on lifting wavelet transform and multi-fractal dimension. Firstly, decompose the vibration signal of bearing by lifting wavelet, select the detail coefficients with better pulse effect to single branch reconstruction [8], to improve the SNR; Then, calculate the multi-fractal dimension of the reconstructed signal, and take it as the state characteristic of bearing 
fault diagnosis, fault diagnosis is completed.

\section{Multi-fractal Dimension}

In this paper, the generalized fractal dimension estimation method based on Renyi entropy will be studied. The thought based on "coverage" is the most convenient for calculation and clear definition in fractal study. Which goes like that: using the closed set $b$ of comparable size to cover the whole set, which scale is $\varepsilon$, requires the number of closed set $b$ is $N(\varepsilon)$. Assuming that the probability of point into $i$ closed set is $P_{i}(\varepsilon)$, then for a given parameter $q$, the formula of generalized information entropy $K_{q}(\varepsilon)$ can be calculated as [9]:

$$
K_{q}(\varepsilon)=\frac{\ln \sum_{i=1}^{N}\left[P_{i}(\varepsilon)\right]^{q}}{1-q}
$$

Then the definition formula of generalized fractal dimension for:

$$
D_{q}=\frac{\lim _{\varepsilon \rightarrow 0} \ln K_{q}(\varepsilon)}{\ln \varepsilon}
$$

So, the multi-fractal dimension can be obtained with the $q$ to change.

When $q=0$, is the box dimension,

$$
D_{0}=\frac{\lim _{\varepsilon \rightarrow 0} \ln N(\varepsilon)}{\ln (1 / \varepsilon)}
$$

When $q=1$, is the information dimension,

$$
D_{1}=\frac{\lim _{\varepsilon \rightarrow 0} \sum_{i} P_{i}(\varepsilon) \ln P_{i}(\varepsilon)}{\ln \varepsilon}
$$

When $q=2$, is the correlation dimension,

$$
D_{2}=\frac{\lim _{\varepsilon \rightarrow 0} \sum_{i} P_{i}(\varepsilon)}{\ln \varepsilon}
$$

Similarly, other dimensions can be obtained by analogy the above formula.

\section{Fault Diagnosis For Rolling Bearing Based on Lifting Wavelet and Multi-fractal Dimension}

The multi-fractal dimension is sensitive to noise as same as the single fractal dimension. Therefore, the desire by multi-fractal dimension to judge bearing working state, must do noise reduction on the vibration signal first. In this paper, the lifting wavelet transform is used to denoise the signal, has the advantages of reconstructing information accurately and real-time. The specific steps are as follows:

(1)Decompose the vibration signal of rolling bearing into three layers by lifting wavelet transform, to obtain the approximation signal and detail coefficients of each layer.

(2)Select the detail coefficients with better pulse effect for single branch reconstruction, and the detail coefficients of other layer will be discarded.

(3)Calculate multi-fractal dimension of the reconstructed signal, and take it as the state characteristic of bearing fault diagnosis. Then, calculate the multi-fractal dimension of bearing original vibration signal, and compare it with the multi-fractal dimension of reconstructed signal. The specific process is shown in Figure 1. 


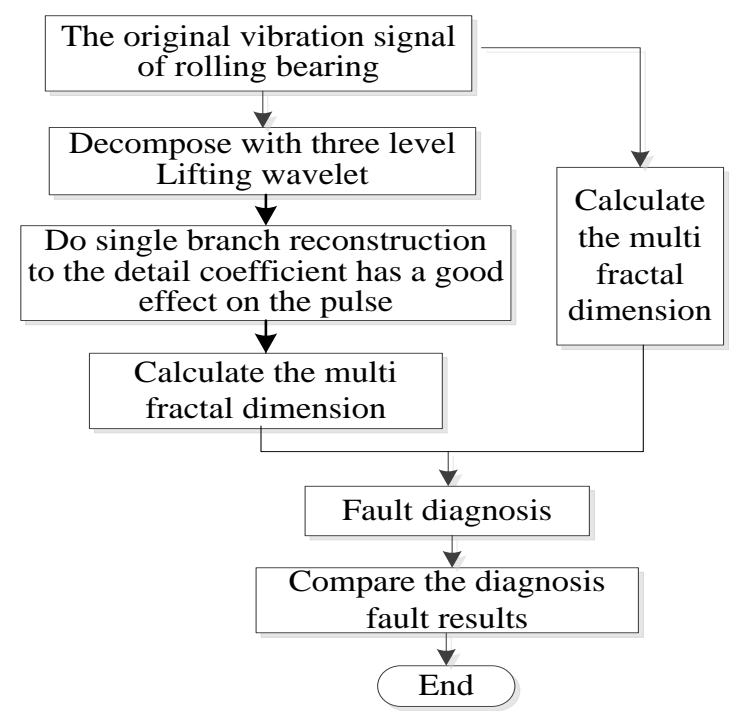

Fig.1. The process of rolling bearing fault diagnosis based on lifting wavelet and multi-fractal dimension

\section{Fault Diagnosis Experiment for Rolling Bearing}

In order to validate the proposed method, do experiment by using the rolling bearing data of Case Western Reserve University in USA [10]. The experimental parameters are as follows, the type is 6205-2RSJEMSKF, the acceleration sensor is installed on the motor drive end, and the no-load speed of the motor is $1797 \mathrm{rpm}$. The data sampling frequency is $12 \mathrm{KHz}$. Respectively select the four states of rolling bearing for experimental analysis such as normal state, rolling element fault, outer ring fault and inner ring fault.

The vibration signal of bearing in normal state, rolling element fault, outer ring fault and inner ring fault are respectively decomposed by three layers lifting wavelet, to obtain the approximation signal and detail coefficients of each layer. The results of decomposition and time domain waveform of rolling bearing in four states as shown in Figure 2 to Figure 5.

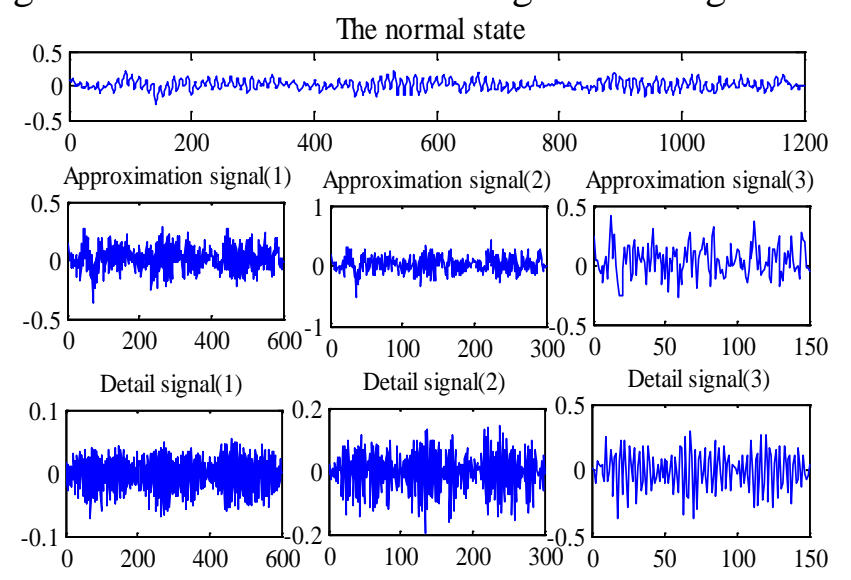

Fig.2. The result of signals in normal decomposed by three layers lifting wavelet 


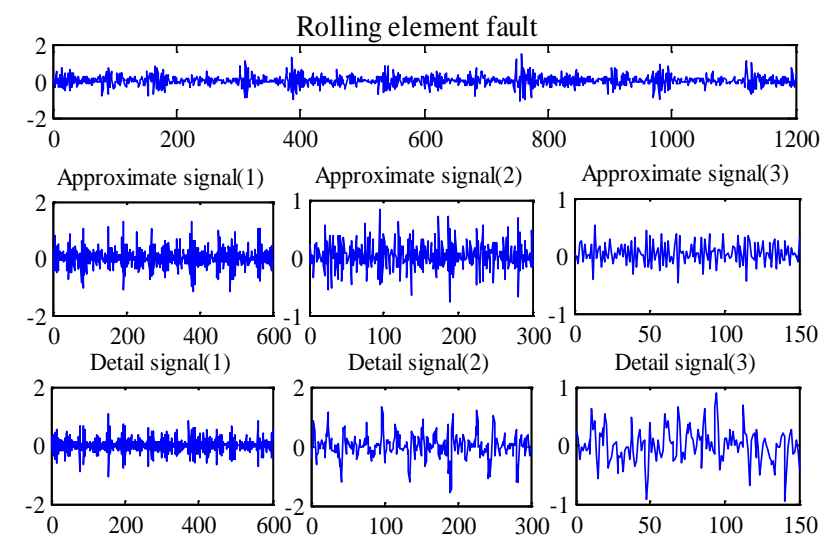

Fig.3. The result of signals with rolling element fault decomposed by three layers lifting wavelet
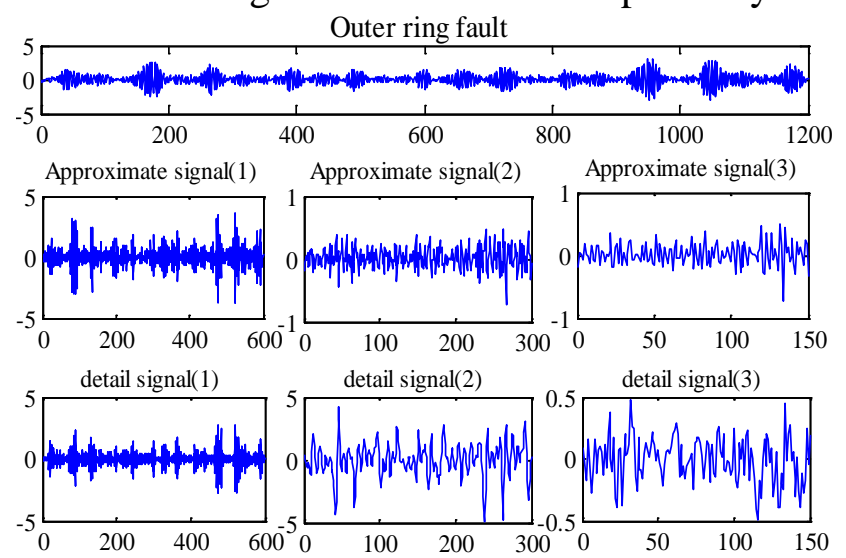

Fig.4. The result of signals with outer ring fault decomposed by three layers lifting wavelet
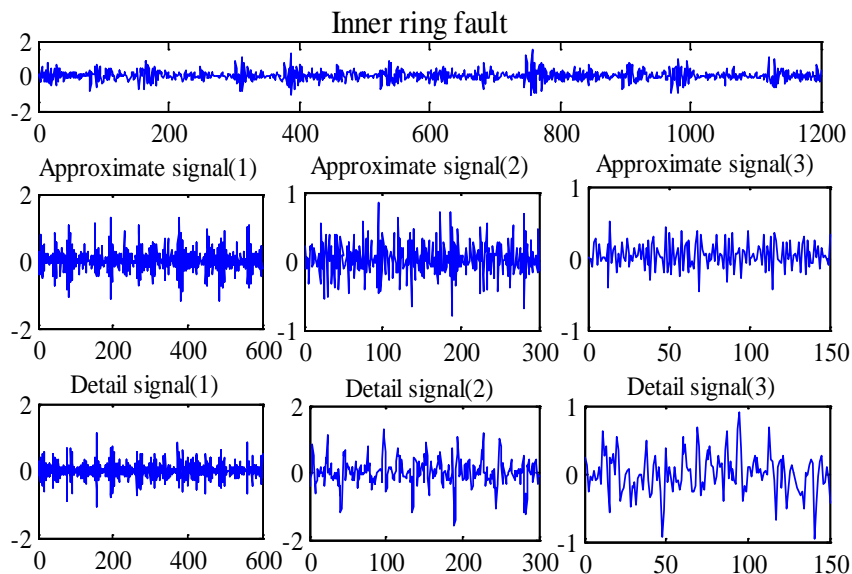

Fig.5. The result of signals with inner ring fault decomposed by three layers lifting wavelet

From Figure 2 to Figure 5 it can be seen that, when rolling bearing in normal, the third detail coefficient of wavelet decomposition has a good extraction effect on pulse; when rolling bearing with rolling element fault, outer ring fault and inner ring fault are all the second layer detail coefficient has a better extraction effect on pulse. Then, respectively do single branch reconstruction to those signal which has a better effect. The results are shown in Figure 6 to Figure 9.
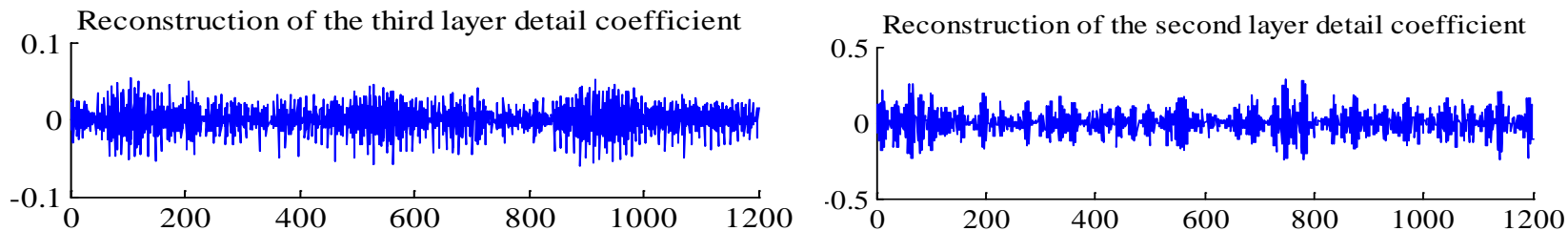

Fig.6. The result of signals in normal reconstructed the second layer detail coefficient by lifting wavelet(left)

Fig.7. The result of signals with rolling element fault reconstructed the second layer detail coefficient by lifting wavelet(right) 

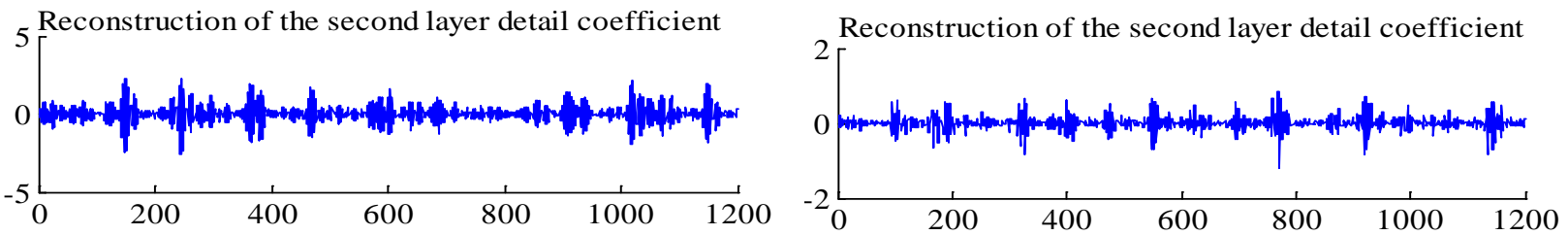

Fig.8. The result of signals with outer ring fault reconstructed the second layer detail coefficient by lifting wavelet(left)

Fig.9. The result of signals with inner ring fault reconstructed the second layer detail coefficient by lifting waveletl(right)

From Figure 6 to Figure 9 it can be seen that, the detail coefficients after single branch reconstruction by lifting wavelet, more clearly reflect the state characteristic of signal, especially the fault impact has becoming evident. Calculate the multi-fractal dimension of these reconstruction detail coefficients, as in table 1 below.

Table1. The multi-fractal dimension of signals in different status reconstructed by lifting wavelet

\begin{tabular}{cccccc}
\hline Status of bearing & $\boldsymbol{D}_{\mathbf{0}}$ & $\boldsymbol{D}_{\mathbf{1}}$ & $\boldsymbol{D}_{\mathbf{2}}$ & $\boldsymbol{D}_{\mathbf{3}}$ & $\boldsymbol{D}_{\mathbf{4}}$ \\
\hline Normal state & 1.7379 & 1.1908 & 1.1017 & 0.9986 & 0.9853 \\
Rolling element fault & 1.6822 & 1.4603 & 1.4496 & 1.3832 & 1.3910 \\
Inner ring fault & 1.6078 & 1.4622 & 1.2115 & 1.1845 & 1.1802 \\
Outer ring fault & 1.6608 & 0.9570 & 0.5461 & 0.5488 & 0.5519
\end{tabular}

It can be seen from table 1, the single fractal dimension can't distinguish of different fault type obviously, but according to the multi-fractal dimension can clearly distinguish each state of rolling bearing. Obviously, the multi-fractal compared to single fractal, describe local details of the signal more comprehensive. For the convenience of comparison, calculate the multi-fractal dimension of the original vibration signal, the results are shown in table 2 . As can be seen, there is tiny difference of fractal dimension among each state, unable to identify all the bearing status. Especially the fractal dimension of inner ring fault and outer ring fault are almost similar, this is because the two exist similar background noise.

Table 2. The multi-fractal dimension of original vibration signals in different status

\begin{tabular}{cccccc}
\hline Status of bearing & $\boldsymbol{D}_{\boldsymbol{0}}$ & $\boldsymbol{D}_{\mathbf{1}}$ & $\boldsymbol{D}_{\mathbf{2}}$ & $\boldsymbol{D}_{\mathbf{3}}$ & $\boldsymbol{D}_{\boldsymbol{4}}$ \\
\hline Normal state & 1.7595 & 1.3402 & 1.2119 & 1.2930 & 1.2816 \\
Rolling element fault & 1.7389 & 1.4790 & 1.4329 & 1.4726 & 1.4687 \\
Inner ring fault & 1.5955 & 1.4873 & 1.4792 & 1.4654 & 1.4630 \\
Outer ring fault & 1.6690 & 1.2035 & 1.0085 & 0.9546 & 0.9688
\end{tabular}

The above experiments indicates, multi-fractal dimension based on lifting wavelet transform has more advantages, can be effectively used for fault diagnosis of rolling bearing.

\section{Conclusions}

Through the proposed method in this paper, by experimenting on rolling bearing vibration signal analysis it can be obtained the beneficial conclusions as follows:

(1)Decompose the original vibration signal of rolling bearing by lifting wavelet transform, and select the detail coefficients with better pulse effect to single branch reconstruction, make the state characteristics of vibration signals more evident.

(2)Compared to the single fractal, using multi-fractal dimension as the characteristics for discriminating the working state of rolling bearing, its principle is clear and concise, description of the local feature of signal more comprehensive.

(3)Combine the lifting wavelet and multi-fractal dimension for rolling bearing fault diagnosis, which can obviously identify all the bearing status. It is a new effective method of fault diagnosis for rolling bearing. 


\section{Acknowledgements}

*Jiande Wu is the corresponding author. This work is supported by National Natural Science Foundation of China (No. 51169007), Science \& Research Program of Yunnan province (No. 2011 CI017 \& 2012CA022 \& 2013DH034), Foundation of Yunnan Education Department (No. 2014J026).

\section{References}

[1] H. R. Patricia, A. Jesus B., and M. A. Ferrer, et al. Application of the Teager-Kaiser energy operator in bearing fault diagnosis, ISA Transaction [J]. 2013 (52) 278-284.

[2] Jin Ma, Zhinong Jiang, Jingji Gao, Feature extraction method based on chaotic fractal theory and its application in fault diagnosis of gas valves, Journal of vibration and shock [J]. 2012 (31) 26-30.

[3] Shiyong Liao, Lin Mi, Xiaohui Shi, Study on Internal Combustion Engine Noise Based on Fractal Theory, Internal combustion engine engineering [J]. 2010 (31) 59-63.

[4] Guangzhong Sun, Fengqin Li, Jianguo Zhang, Study on Plain Bearing Fault Diagnosis Based on Fractal Theory, Coal Mine Machinery [J]. 201031 223-225.

[5] Juntang Chen, Shiyong Liao, Jianfeng Gan, Application of Fractal Dimension to Engine Fault Diagnosis Based on Noise, Vehicle Engine [J]. 2011 (5) 89-92.

[6] Mandelbrot B B, The fractal Gemetry of nature, W H Freeman: San Francisco [M]. 1982 80-89.

[7] Sweldens W, The Lifting Scheme: a Construction of Second Generation Wavelets, SIAM Journal. Math. Anal [J]. 199729 511-546.

[8]Bing Li, PeiLin Zhang, and Dongsheng Liu, Feature Extraction of Roller Bearing Fault Using Morphological Lifting Wavelets, Journal of Vibration, Measurement \& Diagnosis [J]. 201131 36-40.

[9] Grassberger P, Generalized dimensions of strange attractors, Physics Letters A [M]. 1983 227-230.

[10] LoparoK.A. Bearings vibration data set.Case Western Reserve University. <http// www.eecs. cwru.edu/ laboratory/bearing/download.htm>. 